\title{
The therapeutic effect of mineral water from spring 3 in BaileTusnad in experimental alcoholic liver disease in rats - an electron microscopic study
}

Gabriela Dogaru$^{1}$, Ioana Stănescu ${ }^{1}$, Adriana Bulboacă ${ }^{1}$, Marieta Motricală², Vasile Rus ${ }^{3 *}$, Constantin Crăciun ${ }^{4}$, Septimiu Toader ${ }^{5}$, Ioana Adela Rațiu ${ }^{6}$

Corresponding author: Vasile Rus, E-mail: vasile.rus@usamvcluj.ro,

1. "Iuliu Hatieganu” University of Medicine and Pharmacy Cluj-Napoca, Romania , Clinical Rehabilitation Hospital Cluj-Napoca

2. Tușnad Spa Complex - Băile Tușnad, Romania

3. University of Agricultural Sciences and Veterinary Medicine, Cluj-Napoca, Romania

4. Experimental Biology Department, Electron Microscopy Center, "Babeş-Bolyai" University, Cluj-Napoca 5. Experimental Medicine Center, "Iuliu Hatieganu” University of Medicine and Pharmacy Cluj-Napoca

6. University of Oradea, Faculty of Medicine, Medical Sciences Department, Romania

\section{Abstract}

The aim of this study was to investigate by transmission electron microscopy the changes occurring in rat hepatocytes following administration of ethyl alcohol for 70 days, as well as to evaluate the effect of mineral water from spring 3 in Baile Tusnad balneoclimatic resort administered for 30 days after completion of ethyl alcohol administration. After 70 days of ethyl alcohol administration, lipid loading in hepatocytes and a reduction of the rough endoplasmic reticulum were observed. The smooth endoplasmic reticulum was found to be much more abundant and in some hepatocytes that contained high lipid amounts, mitochondria had rarefied matrix and cristae. At 30 days from completion of ethyl alcohol administration, the rats that drank tap water showed progressively decreasing lipid loading. Also, the smooth endoplasmic reticulum was less abundant, while the rough endoplasmic reticulum was more abundant than on day 70 of the experiment. The rats that drank mineral water from spring 3 compared to those that drank tap water over the last 30 days of the experiment had lower hepatocyte lipid loading and the rough endoplasmic reticulum was much more abundant. These ultrastructural differences in the degree of lipid loading and the ratio between the smooth and rough endoplasmic reticulum are due to the beneficial effect of mineral water from spring 3 in Baile Tusnad.

Key words: ethyl alcohol, mineral water, electron microscopy, rat, hepatic steatosis

Introduction Excessive alcohol consumption is a global healthcare problem. The liver sustains the greatest degree of tissue injury by heavy drinking because it is the primary site of ethanol metabolism $[1,2]$. Chronic and excessive alcohol consumption produces a wide spectrum of hepatic lesions, the most characteristic of which are steatosis, hepatitis, and fibrosis/cirrhosis [2, 3, 4].

Ethyl alcohol is mainly metabolized in the liver by hepatocytes, which express high titers of the enzymes involved in alcohol oxidation such as alcohol dehydrogenase (ADH), which is located in the cytosol, and cytochrome P450 2E1 (CYP2E1), which resides in the smooth endoplasmic reticulum [2].

In alcoholic liver disease (ALD), deficiencies of vitamins (A, B1, B6, B9) and minerals (copper, magnesium, selenium, zinc) are frequently found, and these deficiencies influence the pathogenesis of ALD [5].
Experimental studies in rodents have demonstrated that ethyl alcohol administration over long periods leads to a reduction of adipose tissue due to lipolysis $[6,7]$ and to the development of hepatic steatosis $[8,9]$.

The World Health Organization recognizes the therapeutic impact of medicinal mineral waters, and natural mineral water has been considered a curative tool for millennia, having well-known healing properties in terms of prevention, treatment, and rehabilitation of the skin, musculoskeletal, cardiovascular, endocrine, nervous, and other human body systems. Researchers in balneotherapy studies commonly used mineral water with $0.6-31.9 \mathrm{~g} / \mathrm{L}$ total mineralization [10].

Despite some advances in our understanding of the pathogenesis and clinical characteristics of ALD, many questions remain and standardized nomenclature and histological classifications are lacking [4]. 
The aim of this study was to investigate by transmission electron microscopy (TEM) the therapeutic effect of mineral water from spring 3 in Baile Tusnad on hepatocytes following chronic ethyl alcohol administration in rats.

\section{Material and methods}

The study included 25 male Wistar rats with a mean weight of 350 grams, which were fed granular food. The animals were divided into 3 groups: group 1 - negative control (5 animals), group 2 - positive control (6 animals), and group 3 - experimental group (14 animals).

The experiment lasted 100 days and involved 2 stages. The first stage lasted 70 days and was conducted at the Experimental Medicine Center of the University of Medicine and Pharmacy Cluj-Napoca; the second stage lasted 30 days and took place in Baile Tusnad spa resort.

Group 1 animals received tap water, 50-75 $\mathrm{ml} /$ day/animal, throughout the experiment, and group 2 and 3 animals were administered in the first stage of the experiment $12-15 \mathrm{ml}$ of $12 \%$ ethyl alcohol, daily. In the second stage of the experiment, group 2 animals received tap water, 50-75 ml/day/animal, and group 3 animals were administered mineral water from spring 3 in Baile Tusnad, 50-75 ml/day/animal.

According to the analysis report provided by the resort treatment facility, the mineral water from spring 3 in Baile Tusnad has a temperature of $14.5^{\circ} \mathrm{C}$, a pH of 6 and a $\mathrm{CO}_{2}$ content of $1646.0 \mathrm{mg} / \mathrm{l}$. The total mineralization of the water is $3351.0 \mathrm{mg} / \mathrm{l}$ and it contains sodium chloride (chlorine ions $617 \mathrm{mg} / \mathrm{l}$, sodium $288 \mathrm{mg} / \mathrm{l}$ ), calcium $93.8 \mathrm{mg} / \mathrm{l}$, potassium 76 $\mathrm{mg} / \mathrm{l}$, magnesium $35.5 \mathrm{mg} / \mathrm{l}$, iron $17.3 \mathrm{mg} / \mathrm{l}, \mathrm{HCO}_{3}$ $378.2 \mathrm{mg} / \mathrm{l}$ and bromine traces.

At the end of the first stage, 5 animals were euthanized ( 2 animals from group 1, 1 animal from group 2 and 2 animals from group 3) and the other 20 animals were euthanized at the end of the experiment, and $1 \mathrm{~mm}$ thick liver fragments were collected for transmission electron microscopic (TEM) investigations.

The fragments were prefixed with $2.7 \%$ glutaraldehyde in $0.1 \mathrm{M}$ phosphate buffer, for 1.5 hours at $4^{\circ} \mathrm{C}$. After prefixation, they were washed in 4 baths of 1 hour each with $0.15 \mathrm{M}$ phosphate buffer (pH 7.2), and postfixation was performed with $2 \%$ osmic acid in $0.15 \mathrm{M}$ phosphate buffer, for 1 hour at $4^{\circ} \mathrm{C}$. The samples were dehydrated with acetone and embedded in epoxide resin (Epon 812). The blocks obtained were cut with an ultramicrotome (Leica UC 6 ). The sections performed were contrasted with uranyl acetate and lead citrate solutions, examined under the electron microscope (Jeol JEM1010), and photographs were taken.

Throughout the experiment, the animals were kept under standard conditions and euthanasia was carried out according to the legislation in the field (European Directive 63/2010 and Law a43/2014 on the protection of animals used for scientific purposes). This study was performed with the approval of the Sanitary Veterinary and Food Safety Authority Cluj, number 17/ 13.12.2016, and of the Ethics Committee of the University of Medicine and Pharmacy Cluj-Napoca, number 533/23.12.2015.

\section{Results and discussion}

In the control group, in cross section, the majority of the hepatocytes had a round nucleus with 1-2 obvious nucleoli. There were many moderately electron dense spherical or slightly elongated mitochondria, with more or less obvious cristae. The granular endoplasmic reticulum was present in the form of narrow profiles, with numerous attached ribosomes, predominantly located in the perinuclear and perimitochondrial area, in bundles with parallel profiles. The smooth endoplasmic reticulum was mainly found in the outer half of the cytoplasm and was represented by canalicular formations or vesicles. Among mitochondria and GER profiles, numerous glycogen microparticles were present. Lipid inclusions were found in small amounts and in some cells they were lacking (Fig. 1).

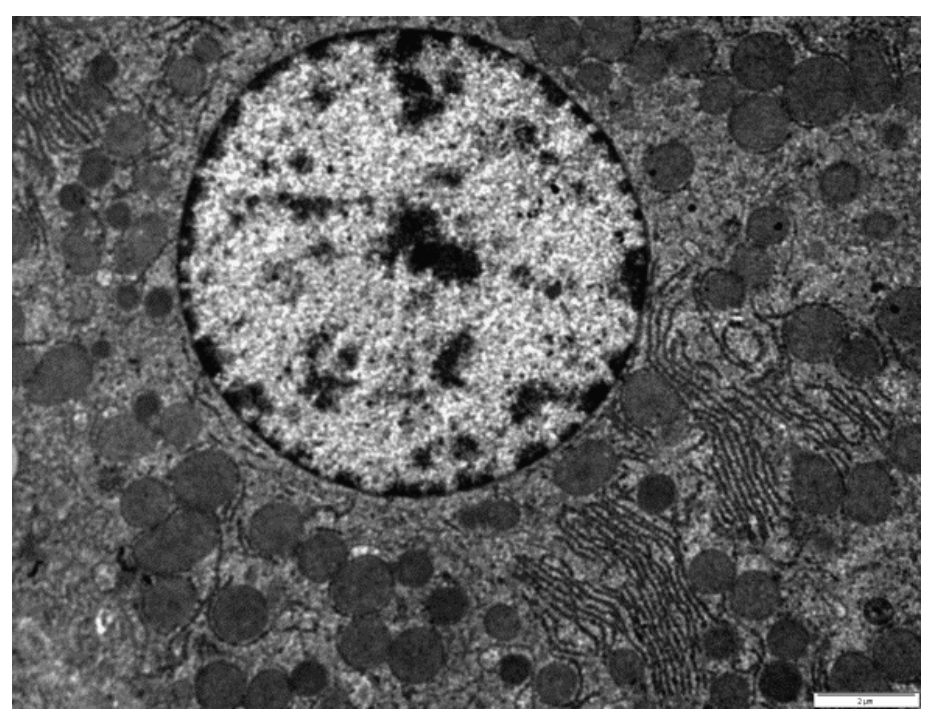

Fig. 1 Liver - group 1 
In the hepatocytes of group 2 and 3 rats, at the end of the first stage of the experiment (day 70), after administration of $12 \%$ ethyl alcohol, no differences were found. In the cytoplasm of hepatocytes, lipid accumulation and a marked decrease of glycogen microparticles were seen. The smooth endoplasmic reticulum was present in the form of many vesicles distributed throughout the cytoplasm and was much more abundant than the rough endoplasmic reticulum, in which a reduction in the number of parallel narrow profiles was found (Fig. 2). In the central area of the liver lobules, some hepatocytes contained large amounts of lipids stored in the cytoplasm. The outline of the nucleus of these hepatocytes was irregular, with a dilated intermembrane space, the mitochondria had rarefied matrix and cristae, and some of them were vacuolized (Fig. 3).

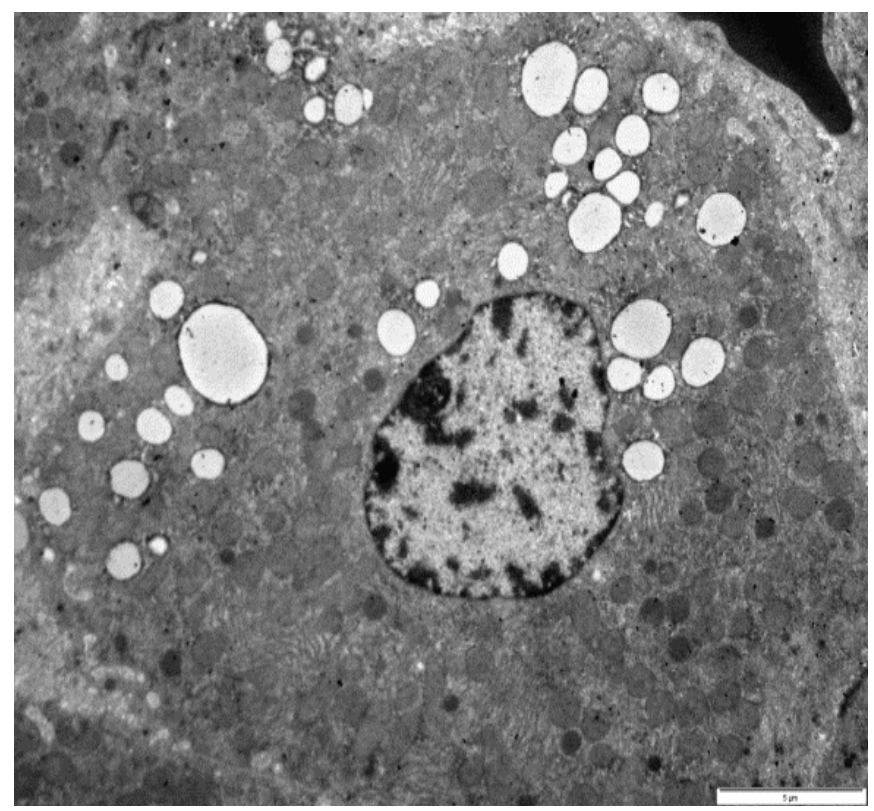

Fig. 2 Appearance of hepatocytes - day 70 of the experiment

In the hepatocytes of group 2 rats, which drank tap water, at the end of the experiment (day 100) there was a moderate decrease in the amount of stored lipids and a moderate increase in the amount of glycogen. The amount of smooth endoplasmic reticulum was reduced. The number of vacuolized mitochondria was also small. An increase in the number and size of lysosomes was observed, as well as abundant narrow profiles of the smooth endoplasmic reticulum (Fig. 4).

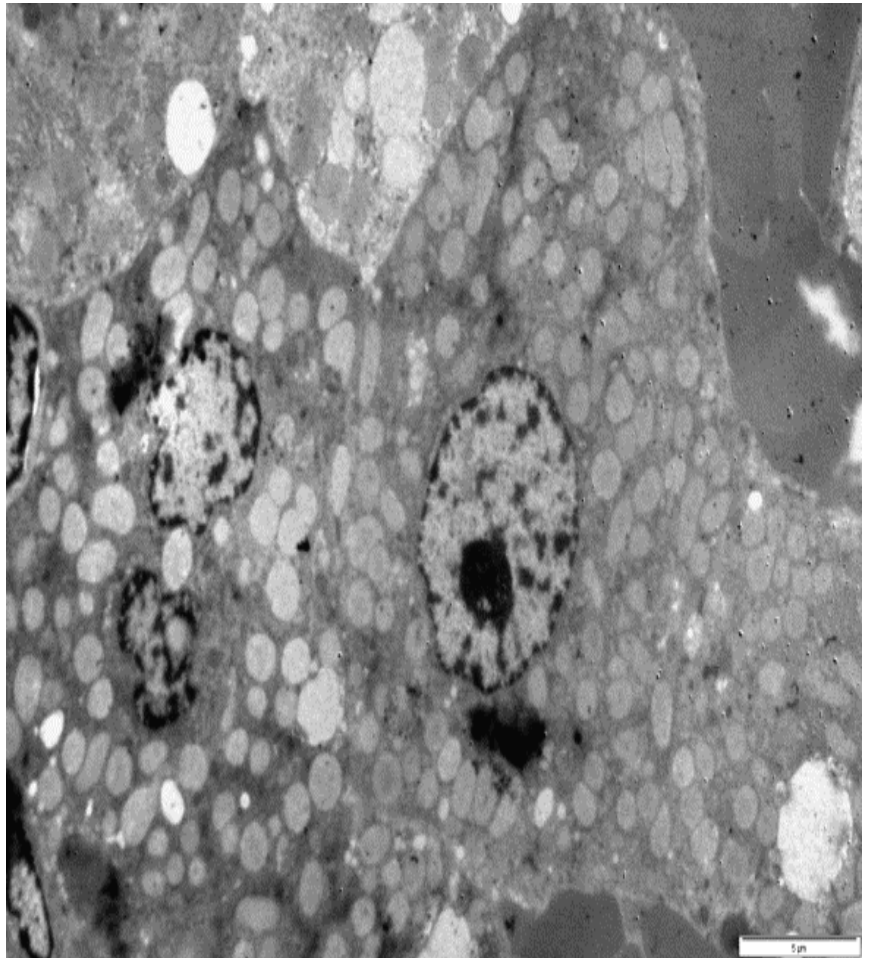

Fig. 3 Appearance of hepatocytes in the centrilobular area - day 70 of the experiment

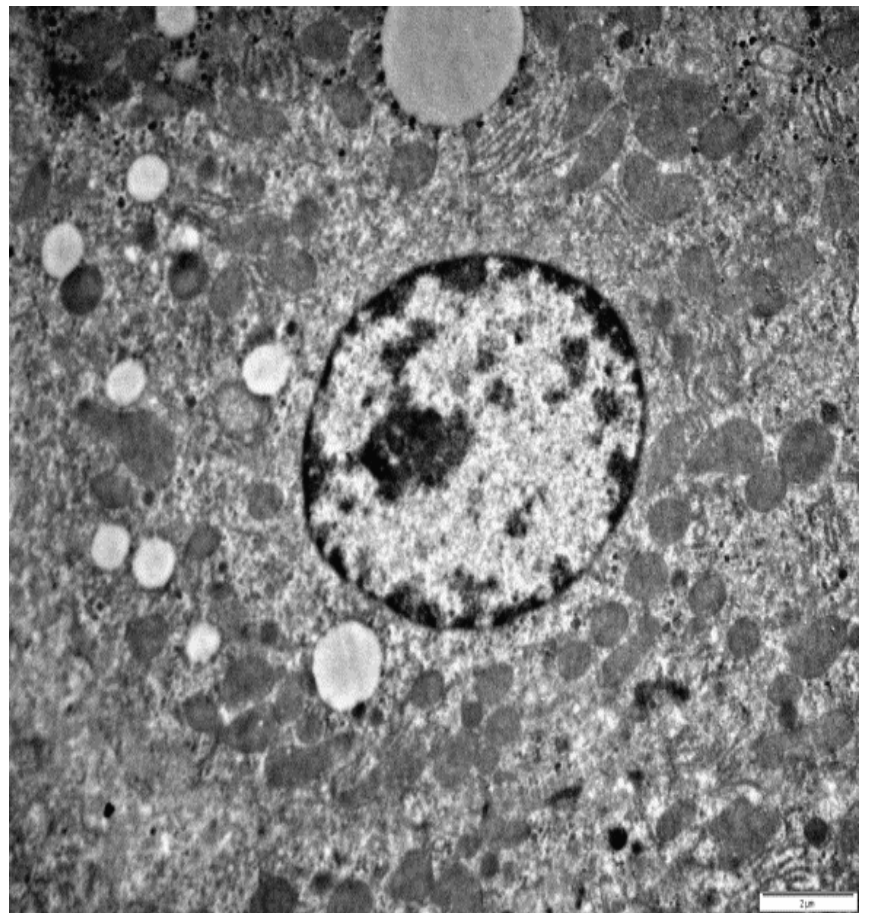

Fig. 4 Appearance of hepatocytes in group 2 day 100 of the experiment

At the end of the experiment (day 100), in the hepatocytes of group 3 rats, who drank mineral water from spring 3 in Baile Tusnad, there was a marked decrease in lipid loading and an increase in the amount of glycogen. Also, abundant lysosomes and 
rough endoplasmic reticulum were observed in the cytoplasm, while the amount of smooth endoplasmic reticulum was reduced. The profiles of the rough endoplasmic reticulum were dense and parallel, and the smooth endoplasmic reticulum was present in the form of vesicles (Fig. 5).

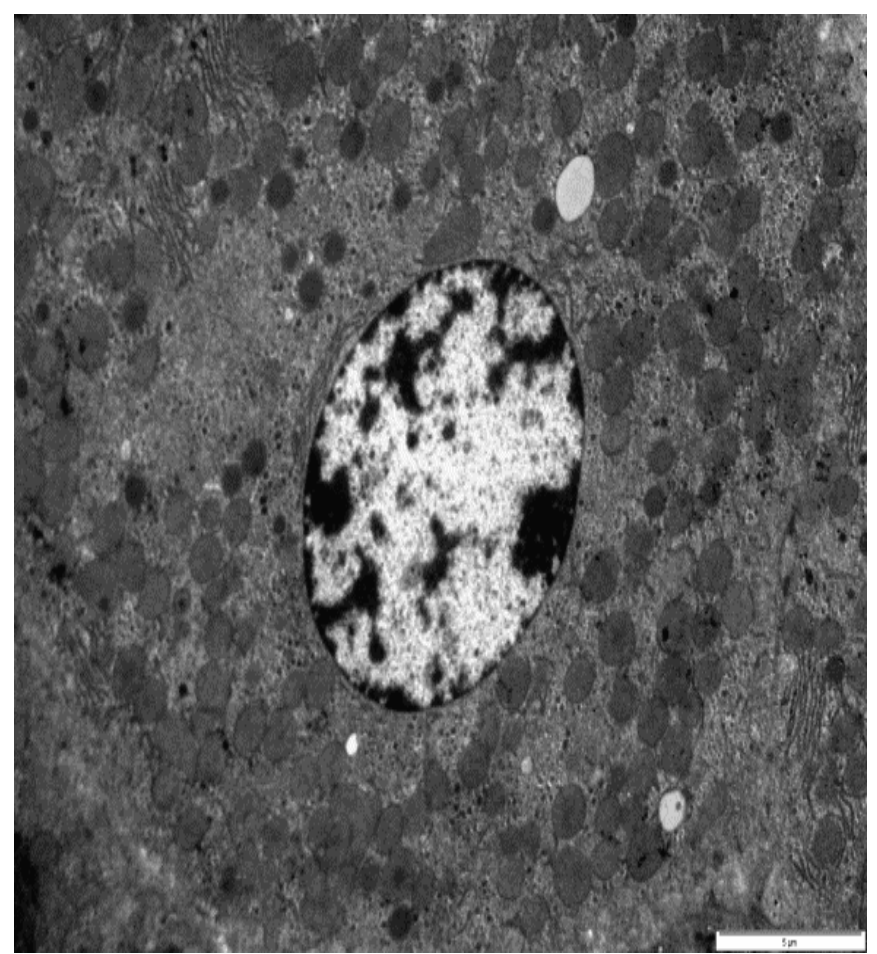

Fig. 5 Appearance of hepatocytes in group 3 day 100 of the experiment

Administration of ethyl alcohol for 70 days led to hepatic steatosis with intracytoplasmic lipid accumulation. The amount of lipids accumulated in the cytoplasm of hepatocytes was higher in the cells around the centrilobular venule.

The increase in the amount of lipids led to a change in the ratio between the rough and smooth endoplasmic reticulum, so that at the end of the first stage of the experiment, the smooth endoplasmic reticulum was much more abundant in hepatocytes, suggesting the fact that cell metabolism largely involved lipids to the detriment of protein biosynthesis taking place in the rough endoplasmic reticulum. Similar results referring to ultrastructural changes occurring in hepatocytes after ethyl alcohol administration in rats were reported by other authors $[11,12,13]$.

At the end of the experiment, the amount of lipids stored in the cytoplasm of hepatocytes in group 2 rats was smaller than on day 70 of the experiment.
It was also found that the smooth endoplasmic reticulum was less abundant, while the rough endoplasmic reticulum became increasingly abundant, but far from being as abundant as in group 1.

At the end of the second stage of the experiment, microstructure differences were observed between the hepatocytes of group 2 and 3 rats. Thus, in group 2, the amount of smooth endoplasmic reticulum as well as lipid loading in hepatocytes was higher than in group 3. The high amount of smooth endoplasmic reticulum present in the cytoplasm of hepatocytes seems to be related to lipid metabolization by cytochrome P450 2E1, as it is known that reactions catalyzed by cytochrome P450 2E1 take place in the lumen of the smooth endoplasmic reticulum [2].

These differences in the degree of lipid loading as well as the ratio between the smooth and rough endoplasmic reticulum are due to the beneficial effect of mineral water from spring 3 in Baile Tusnad. Similar results regarding the degree of lipid loading in hepatocytes were described in other optical microscopic studies $[8,9]$. Higher hepatocyte lipid loading in the centrilobular area was also reported by other authors $[2,14]$. The rarefied appearance of the matrix and mitochondrial cristae, as well as the ballooned appearance of mitochondria also seems to be related to lipid metabolization in hepatocytes. Some authors [2] maintain that one way by which hepatocytes minimize the toxicity of acetaldehyde is by rapidly oxidizing it to acetate using the enzyme aldehyde dehydrogenase 2 (ALDH2) inside mitochondria.

\section{Conclusions}

Ethyl alcohol administration in rats for 70 days induced lipid accumulation in the cytoplasm of hepatocytes, a decrease in the amount of rough endoplasmic reticulum, an increase in the amount of smooth endoplasmic reticulum, while mitochondria had rarefied cristae and matrix.

At 30 days from cessation of ethyl alcohol administration, the rats that drank tap water during this period showed a reduction in the amount of lipids stored in hepatocytes and a decrease in the amount of smooth endoplasmic reticulum, accompanied by an increase in the amount of rough endoplasmic reticulum. 
The rats that received mineral water from spring 3 in Baile Tusnad for 30 days after cessation of ethyl alcohol administration had a lower degree of lipid loading and a smaller amount of smooth endoplasmic reticulum, while the rough endoplasmic reticulum was more abundant compared to rats that drank tap water during this period. These differences are due to the curative and therapeutic effect of the mineral water from spring 3 in Baile Tusnad.

\section{Bibliography}

1. Barve S., S.Y. Chen, I. Kirpich, W.H. Watson, C. McClain. Development, Prevention, and Treatment of Alcohol-Induced Organ Injury. Alcohol Research: Current Reviews, 38(2): e1-e-14, 2017.

2. Osna N.A., T.M. Donohue, K.K. Kharbanda. Alcoholic Liver Disease: Pathogenesis and Current Management. Alcohol Research: Current Reviews, 38(2):7-21, 2017.

3. Bruha R., K. Dvorak, J. Petrtyl. Alcoholic liver disease. World J Hepatol, 4(3): 81-90, 2012, doi: 10.4254/wjh.v4.i3.81.

4. Orman E.S., G. Odena, R. Bataller. Alcoholic liver disease: Pathogenesis, management, and novel targets for therapy. J Gastroenterol Hepatol, 28(0 1): 77-84, 2013.

5. Halsted C.H. Nutrition and alcoholic liver disease. Seminars in Liver Disease, 24(3):289-304, 2004.

6. Wang Z.G., X.B. Dou, Z.X. Zhou, Z.Y. Song. Adipose tissue-liver axis in alcoholic liver disease. World Journal of Gastrointestinal Pathophysiology, 7(1):17-26, 2016.

7. Wei X, Shi X, Zhong W, Zhao Y, Tang Y, et al. (2013) Chronic Alcohol Exposure Disturbs Lipid Homeostasis at the Adipose TissueLiver Axis in Mice: Analysis of Triacylglycerols Using High-Resolution Mass Spectrometry in Combination with In Vivo Metabolite Deuterium Labeling. PLoS ONE 8(2): e55382. doi:10.1371/journal.pone.0055382.

8. Dogaru G., Motricală M., Molnár Á., Rus V. An experimental study regarding the biological effects of mineral water from spring 3 in Băile Tuşnad on some organs after ethyl alcohol administration. Balneo Research
Journal, $\quad 7(1), \quad 23-28, \quad 2016$, DOI: http://dx.doi.org/10.12680/balneo.2016.116.

9. Dogaru G., M. Motricală, Á. Molnár, V. Rus. Effects of mineral water from spring 3 in Băile Tuşnad on experimentally induced alcoholic liver disease. Balneo Research Journal, 8(3), 125-128, 2017, DOI: http://dx.doi.org/10.12680/balneo.2017.147

10. Rapoliene L., A. Razbadauskas, J. Sąlyga, A. Martinkėnas. Stress and Fatigue Management Using Balneotherapy in a Short-Time Randomized Controlled Trial. EvidenceBased Complementary and Alternative Medicine, Volume 2016, Article ID 9631684, 10 http://dx.doi.org/10.1155/2016/9631684. pages

11. Matei E., S. Zamfirescu, A. Anghel. Electron Microscopy observations of the hepatic tissue ultrastructure after the acute intoxication with ethanol at rats. Annals of the Romanian Society for Cell Biology 15(1):105-112, 2010.

12. Pribac G., C. Craciun, C. Rosioru, A. Ardelean, M Czapar, I. Simeoni, L. Mos. Ultrastructural studies on the hepatoprotective effects of Trigonella foenum graecum seeds in alcoholic rats. Annals of RSCB, XV(2):373389, 2010.

13. Roşioru C., G. Pribac, I. Simeoni, C. Crăciun, A. Ardelean. Trigonella foenum graecum seeds - a natural hepatoprotector which prevents ehanol-induced toxicity. Annals of RSCB, XV(2):390-399, 2010.

14. Theise N.D. Histopathology of Alcoholic Liver Disease. Clinical Liver Disease, 2(2): 64-67, 2013. 